\title{
Specific growth rate and carbon sugar consumption of diazotrophs isolated from rice rhizosphere
}

\begin{abstract}
A study was conducted in in vitro condition to determine the specific growth rate, sugar consumption and generation time of four diazotrophic bacterial species, Burkholderia, Rhizobium and two Corynebacterium sp. in four different carbon substrates. Growth of all bacterial strains was significantly affected by the sugars. Burkholderia sp. (Sb13) rapidly attained maximum population in glucose, fructose and sucrose and the specific growth rate best fitted with exponential model. The cell generation times of Burkholderia sp. (Sb13) in glucose, fructose and sucrose substrate were $0.4,0.55$ and $0.1 \mathrm{~h}$, respectively. Rhizobium sp. (Sb16) followed an exponential growth model in fructose and its generation time was $0.62 \mathrm{~h}$. The growth of Corynebacterium sp. (Sb26) was higher in arabinose and followed logarithmic growth model with generation time of $0.68 \mathrm{~h}$. Corynebacterium sp. (Sb35) showed faster generation time $(1 \mathrm{~h})$ in sucrose substrates and the specific growth rate in all four carbon substrates followed logarithmic model of growth phase. Burkholderia sp. and Corynebacterium sp. (Sb26) consumed $100 \%$ of arabinose after $36 \mathrm{~h}$ of incubation period. The total sugar consumption by the diazotrophs were higher in logarithmic model of growth phase compared to exponential, power and polynomial model.
\end{abstract}

Keyword: Carbon sugars; Generation time; Specific growth rate; Substrate consumption 